Finite Fields Appl. 64 (2020), Article 101672.

\title{
ON SOME DETERMINANTS INVOLVING JACOBI SYMBOLS
}

\author{
DMITRY KRACHUN, FEDOR PETROV, ZHI-WEI SUN, MAXIM VSEMIRNOV
}

\begin{abstract}
In this paper we study some conjectures on determinants with Jacobi symbol entries posed by Z.-W. Sun. For any positive integer $n \equiv 3(\bmod 4)$, we show that
\end{abstract}

$$
(6,1)_{n}=[6,1]_{n}=(3,2)_{n}=[3,2]_{n}=0
$$

and

$$
(4,2)_{n}=(8,8)_{n}=(3,3)_{n}=(21,112)_{n}=0
$$

as conjectured by Sun, where

$$
(c, d)_{n}=\left|\left(\frac{i^{2}+c i j+d j^{2}}{n}\right)\right|_{1 \leqslant i, j \leqslant n-1}
$$

and

$$
[c, d]_{n}=\left|\left(\frac{i^{2}+c i j+d j^{2}}{n}\right)\right|_{0 \leqslant i, j \leqslant n-1}
$$

with $(\dot{\dot{n}})$ the Jacobi symbol. We also prove that $(10,9)_{p}=0$ for any prime $p \equiv 5(\bmod 12)$, and $[5,5]_{p}=0$ for any prime $p \equiv 13,17(\bmod 20)$, which were also conjectured by Sun. Our proofs involve character sums over finite fields.

\section{INTRODUCTION}

For an $n \times n$ matrix $\left[a_{i j}\right]_{1 \leqslant i, j \leqslant n}$ over a field, we simply denote its determinant by $\left|a_{i j}\right|_{1 \leqslant i, j \leqslant n}$. In this paper we study some conjectures on determinants with Jacobi symbol entries posed by Z.-W. Sun [11].

Let $p$ be an odd prime. In 2004, R. Chapman [2] determined the values of

$$
\left|\left(\frac{i+j-1}{p}\right)\right|_{1 \leqslant i, j \leqslant(p-1) / 2}=\left(\frac{-1}{p}\right)\left|\left(\frac{i+j}{p}\right)\right|_{1 \leqslant i, j \leqslant(p-1) / 2}
$$

and

$$
\left|\left(\frac{i+j-1}{p}\right)\right|_{1 \leqslant i, j \leqslant(p+1) / 2}=\left|\left(\frac{i+j}{p}\right)\right|_{0 \leqslant i, j \leqslant(p-1) / 2},
$$

Key words and phrases. Determinants, Jacobi symbols, character sums over finite fields. 2020 Mathematics Subject Classification. Primary 11C20, 11T24; Secondary 11E16, $15 \mathrm{~A} 15$.

The work is supported by the NSFC (Natural Science Foundation of China)-RFBR (Russian Foundation for Basic Research) Cooperation and Exchange Program (grants NSFC 11811530072 and RFBR 18-51-53020-GFEN-a). The third author is also supported by the Natural Science Foundation of China (grant 11971222). 
where $(\dot{\bar{p}})$ denotes the Legendre symbol. Chapman's conjecture on the evaluation of

$$
\left|\left(\frac{j-i}{p}\right)\right|_{0 \leqslant i, j \leqslant(p-1) / 2}
$$

was confirmed by M. Vsemirnov $[12,13]$ via matrix decomposition. With this background, Z.-W. Sun [11] studied some new kinds of determinants with Legendre symbol or Jacobi symbol entries.

For any odd integer $n>1$ and integers $c$ and $d$, Sun [11] introduced the notations

$$
(c, d)_{n}:=\left|\left(\frac{i^{2}+c i j+d j^{2}}{n}\right)\right|_{1 \leqslant i, j \leqslant n-1}
$$

and

$$
[c, d]_{n}:=\left|\left(\frac{i^{2}+c i j+d j^{2}}{n}\right)\right|_{0 \leqslant i, j \leqslant n-1},
$$

where $(\dot{\bar{n}})$ denotes the Jacobi symbol. He showed that

$$
\left(\frac{d}{n}\right)=-1 \Rightarrow(c, d)_{n}=0
$$

and that for any odd prime $p$ we have

$$
\left(\frac{d}{p}\right)=1 \Rightarrow[c, d]_{p}= \begin{cases}\frac{p-1}{2}(c, d)_{p} & \text { if } p \nmid c^{2}-4 d, \\ \frac{1-p}{p-2}(c, d)_{p} & \text { if } p \mid c^{2}-4 d .\end{cases}
$$

For $a \in \mathbb{Z}$ and $n \in \mathbb{Z}^{+}=\{1,2,3, \ldots\}$, if $a$ is relatively prime to $n$ and $x^{2} \equiv a(\bmod n)$ for some $x \in \mathbb{Z}$, then $a$ is called a quadratic residue modulo $n$. If $n$ is odd and $a$ is a quadratic residue modulo $n$, then $\left(\frac{a}{n}\right)=1$ since $a$ is a quadratic residue modulo any prime divisor of $n$.

Now we state our first theorem.

Theorem 1.1. Let $n>1$ be an odd integer.

(i) If -1 is not a quadratic residue modulo $n$, then

$$
(6,1)_{n}=(3,2)_{n}=0 \text { and }[6,1]_{n}=[3,2]_{n}=0 .
$$

(ii) If -2 is not a quadratic residue modulo $n$, then

$$
(4,2)_{n}=(8,8)_{n}=0 \text { and }[4,2]_{n}=[8,8]_{n}=0 .
$$

(iii) If -3 is not a quadratic residue modulo $n$, then

$$
(3,3)_{n}=(6,-3)_{n}=0 \text { and }[3,3]_{n}=[6,-3]_{n}=0 .
$$

(iv) If -7 is not a quadratic residue modulo $n$, then

$$
(21,112)_{n}=(42,-7)_{n}=0 \text { and }[21,112]_{n}=[42,-7]_{n}=0 .
$$

Combining Theorem 1.1 with (1.3), we immediately obtain the following consequence which was conjectured by Sun [11, Conjecture 4.8(ii)]. 
Corollary 1.1. For any positive integer $n \equiv 3(\bmod 4)$, we have

$$
(6,1)_{n}=[6,1]_{n}=(3,2)_{n}=[3,2]_{n}=0
$$

and

$$
(4,2)_{n}=(8,8)_{n}=(3,3)_{n}=(21,112)_{n}=0 .
$$

Actually we deduce Theorem 1.1 from the following theorems.

Theorem 1.2. Let $n$ be a positive odd integer which is squarefree. For any $c, d, i \in \mathbb{Z}$, we have

$$
\sum_{j=0}^{n-1}\left(\frac{j}{n}\right)\left(\frac{i^{2}+c i j+d j^{2}}{n}\right)=\sum_{j=0}^{n-1}\left(\frac{-j}{n}\right)\left(\frac{i^{2}+2 c i j+\left(c^{2}-4 d\right) j^{2}}{n}\right) .
$$

Theorem 1.3. Let $n$ be a positive odd integer which is squarefree, and let $i \in \mathbb{Z}$. Then

$$
\begin{aligned}
\sum_{j=0}^{n-1}\left(\frac{j}{n}\right)\left(\frac{i^{2}+3 i j+2 j^{2}}{n}\right) & =0 \quad \text { if }-1 R n \text { fails, } \\
\sum_{j=0}^{n-1}\left(\frac{j}{n}\right)\left(\frac{i^{2}+4 i j+2 j^{2}}{n}\right) & =0 \quad \text { if }-2 R n \text { fails, } \\
\sum_{j=0}^{n-1}\left(\frac{j}{n}\right)\left(\frac{i^{2}+3 i j+3 j^{2}}{n}\right) & =0 \quad \text { if }-3 R n \text { fails, } \\
\sum_{j=0}^{n-1}\left(\frac{j}{n}\right)\left(\frac{i^{2}+21 i j+112 j^{2}}{n}\right) & =0 \quad \text { if }-7 R n \text { fails, }
\end{aligned}
$$

where the notation $m R n$ means that $m$ is a quadratic residue modulo $n$.

Our following result was originally conjectured by Sun [11, Conjecture 4.8(iv)].

Theorem 1.4. (i) $(10,9)_{p}=0$ for any prime $p \equiv 5(\bmod 12)$.

(ii) $[5,5]_{p}=0$ for any prime $p \equiv 13,17(\bmod 20)$.

In fact, our proof of Theorem 1.4 yields a stronger result: For each integer $y$, we have

$$
\sum_{x=0}^{p-1}\left(\frac{x^{5}+10 x^{3} y+9 x y^{2}}{p}\right)=0
$$

for any prime $p \equiv 5(\bmod 12)$, and

$$
\sum_{x=0}^{p-1}\left(\frac{x^{5}+5 x^{3} y+5 x y^{2}}{p}\right)=0
$$

for any prime $p \equiv 13,17(\bmod 20)$.

We will prove Theorem 1.2, Theorems 1.3 and 1.1, and Theorem 1.4 in Sections 2-4 respectively. 
Sun $\left[11\right.$, Conjecture 4.8(iv)] also conjectured that $(8,18)_{p}=[8,18]_{p}=0$ for any prime $p \equiv 13,17(\bmod 24)$. Moreover, Sun [10] conjectured that

$$
\sum_{x=0}^{p-1}\left(\frac{x^{5}+8 x^{3} y+18 x y^{2}}{p}\right)=0
$$

for any prime $p \equiv 13,17(\bmod 24)$ and integer $y$, and this was confirmed by M. Stoll via two elliptic curves with complex multiplication by $\mathbb{Z}[\sqrt{-6}]$ (see the answer in $[10])$.

For any prime $p \equiv 1(\bmod 4)$ and $a, b, c \in \mathbb{Z}$, we provide in Section 5 a sufficient condition for

$$
\sum_{x=0}^{p-1}\left(\frac{a x^{5}+b x^{3}+c x}{p}\right)=2 \sum_{x=1}^{(p-1) / 2}\left(\frac{x}{p}\right)\left(\frac{a\left(x^{2}\right)^{2}+b x^{2}+c}{p}\right)=0 .
$$

\section{Proof of Theorem 1.2}

Lemma 2.1. Let $p$ be an odd prime and let $c, d, i \in \mathbb{Z}$ with $p \nmid c$. Then

$$
\sum_{j=0}^{p-1}\left(\frac{j}{p}\right)\left(\frac{i^{2}+c i j+d j^{2}}{p}\right) \equiv-\left(\frac{c i}{p}\right) \sum_{k=0}^{p-1}\left(\begin{array}{c}
4 k \\
2 k
\end{array}\right)\left(\begin{array}{c}
2 k \\
k
\end{array}\right)\left(\frac{d}{16 c^{2}}\right)^{k}(\bmod p) .
$$

Proof. If $p \mid i$, then both sides of the congruence (2.1) are zero.

Below we assume $p \nmid i$ and let $L$ denote the left-hand side of the congruence (2.1). As $\{i r: r=0, \ldots, p-1\}$ is a complete system of residues modulo $p$, we have

$$
\begin{aligned}
L & =\sum_{r=0}^{p-1}\left(\frac{i r}{p}\right)\left(\frac{i^{2}+c i(i r)+d(i r)^{2}}{p}\right)=\left(\frac{i^{3}}{p}\right) \sum_{r=0}^{p-1}\left(\frac{r}{p}\right)\left(\frac{1+c r+d r^{2}}{p}\right) \\
& \equiv\left(\frac{i}{p}\right) \sum_{r=1}^{p-1} r^{(p-1) / 2}\left(1+c r+d r^{2}\right)^{(p-1) / 2} \\
& \equiv\left(\frac{i}{p}\right) \sum_{r=1}^{p-1}\left(r^{-1}+c+d r\right)^{(p-1) / 2}(\bmod p) .
\end{aligned}
$$

We may write $\left(x^{-1}+c+d x\right)^{(p-1) / 2}=\sum_{s=-(p-1) / 2}^{(p-1) / 2} a_{s} x^{s}$ with $a_{s} \in \mathbb{Z}$. For any integer $s$, it is well known (cf. [4, p. 235]) that

$$
\sum_{r=1}^{p-1} r^{s} \equiv \begin{cases}-1(\bmod p) & \text { if } p-1 \mid s \\ 0(\bmod p) & \text { otherwise }\end{cases}
$$

Therefore,

$$
\sum_{r=1}^{p-1}\left(r^{-1}+c+d r\right)^{(p-1) / 2}=\sum_{s=-(p-1) / 2}^{(p-1) / 2} a_{s} \sum_{r=1}^{p-1} r^{s} \equiv-a_{0}(\bmod p) .
$$


Clearly,

$$
\begin{aligned}
a_{0} & =\sum_{k=0}^{(p-1) / 2}\left(\begin{array}{c}
(p-1) / 2 \\
2 k
\end{array}\right)\left(\begin{array}{c}
2 k \\
k
\end{array}\right) c^{(p-1) / 2-2 k} d^{k} \\
& \equiv \sum_{k=0}^{(p-1) / 2}\left(\begin{array}{c}
-1 / 2 \\
2 k
\end{array}\right)\left(\begin{array}{c}
2 k \\
k
\end{array}\right)\left(\frac{c}{p}\right)\left(\frac{d}{c^{2}}\right)^{k}=\left(\frac{c}{p}\right) \sum_{k=0}^{(p-1) / 2} \frac{\left(\begin{array}{c}
4 k \\
2 k
\end{array}\right)\left(\begin{array}{c}
2 k \\
k
\end{array}\right)}{(-4)^{2 k}}\left(\frac{d}{c^{2}}\right)^{k} \\
& =\left(\frac{c}{p}\right) \sum_{k=0}^{p-1}\left(\begin{array}{c}
4 k \\
2 k
\end{array}\right)\left(\begin{array}{c}
2 k \\
k
\end{array}\right)\left(\frac{d}{16 c^{2}}\right)^{k}(\bmod p) .
\end{aligned}
$$

So, by the above, we finally obtain (2.1).

Lemma 2.2. Let $p$ be any odd prime. Then we have the congruence

$$
\sum_{k=0}^{p-1} \frac{\left(\begin{array}{c}
4 k \\
2 k
\end{array}\right)\left(\begin{array}{c}
2 k \\
k
\end{array}\right)}{64^{k}}\left(x^{k}-\left(\frac{-2}{p}\right)(1-x)^{k}\right) \equiv 0\left(\bmod p^{2-\delta_{p, 3}}\right)
$$

in the ring $\mathbb{Z}_{p}[x]$, where $\mathbb{Z}_{p}$ is the ring of all $p$-adic integers, and $\delta_{p, 3}$ is 1 or 0 according as $p=3$ or not.

Remark 2.1. For any prime $p>3$, the congruence (2.3) is due to Sun $[9$, (1.15)]. We can easily verify that (2.3) also holds for $p=3$.

Proof of Theorem 1.2. Clearly both sides of (1.5) vanish if $n=1$. Below we assume $n>1$ and distinguish three cases.

Case 1. $n$ is an odd prime $p$.

Define

$$
D:=\sum_{j=0}^{p-1}\left(\frac{j}{p}\right)\left(\frac{i^{2}+c i j+d j^{2}}{p}\right)-\sum_{j=0}^{p-1}\left(\frac{-j}{p}\right)\left(\frac{i^{2}+2 c i j+\left(c^{2}-4 d\right) j^{2}}{p}\right) .
$$

If $p \mid c$ and $p \equiv 3(\bmod 4)$, then

$$
\begin{aligned}
D= & \sum_{j=1}^{(p-1) / 2}\left(\left(\frac{j}{p}\right)+\left(\frac{p-j}{p}\right)\right)\left(\frac{i^{2}+d j^{2}}{p}\right) \\
& -\sum_{j=1}^{(p-1) / 2}\left(\left(\frac{-j}{p}\right)+\left(\frac{-(p-j)}{p}\right)\right)\left(\frac{i^{2}-4 d j^{2}}{p}\right) \\
= & 0-0=0 .
\end{aligned}
$$

When $p \mid c$ and $p \equiv 1(\bmod 4)$, for $q=((p-1) / 2) !$ we have $q^{2} \equiv-1(\bmod p)$ and $\left(\frac{2 q}{p}\right)=1$ (cf. [11, Remark 1.1 and Lemma 2.3]), thus

$$
D=\sum_{j=1}^{p-1}\left(\frac{j}{p}\right)\left(\frac{i^{2}+d j^{2}}{p}\right)-\sum_{j=1}^{p-1}\left(\frac{-j}{p}\right)\left(\frac{i^{2}-4 d j^{2}}{p}\right)
$$




$$
=\sum_{j=1}^{p-1}\left(\frac{2 q j}{p}\right)\left(\frac{i^{2}+d(2 q j)^{2}}{p}\right)-\sum_{j=1}^{p-1}\left(\frac{j}{p}\right)\left(\frac{i^{2}-4 d j^{2}}{p}\right)=0 .
$$

Now suppose that $p \nmid c$. By Lemma 2.2,

$$
\begin{aligned}
& \sum_{k=0}^{p-1}\left(\begin{array}{c}
4 k \\
2 k
\end{array}\right)\left(\begin{array}{c}
2 k \\
k
\end{array}\right)\left(\frac{d}{16 c^{2}}\right)^{k} \\
= & \sum_{k=0}^{p-1} \frac{\left(\begin{array}{c}
4 k \\
2 k
\end{array}\right)\left(\begin{array}{c}
2 k \\
k
\end{array}\right)}{64^{k}}\left(\frac{4 d}{c^{2}}\right)^{k} \\
\equiv & \left(\frac{-2}{p}\right) \sum_{k=0}^{p-1} \frac{\left(\begin{array}{c}
4 k \\
2 k
\end{array}\right)\left(\begin{array}{c}
2 k \\
k
\end{array}\right)}{64^{k}}\left(1-\frac{4 d}{c^{2}}\right)^{k} \\
= & \left(\frac{-2}{p}\right) \sum_{k=0}^{p-1}\left(\begin{array}{c}
4 k \\
2 k
\end{array}\right)\left(\begin{array}{c}
2 k \\
k
\end{array}\right)\left(\frac{c^{2}-4 d}{16(2 c)^{2}}\right)^{k}\left(\bmod p^{2-\delta_{p, 3}}\right) .
\end{aligned}
$$

Combining this with Lemma 2.1, we obtain that

$$
\begin{aligned}
& \sum_{j=0}^{p-1}\left(\frac{j}{p}\right)\left(\frac{i^{2}+c i j+d j^{2}}{p}\right) \\
\equiv & -\left(\frac{-2 c i}{p}\right) \sum_{k=0}^{p-1}\left(\begin{array}{c}
4 k \\
2 k
\end{array}\right)\left(\begin{array}{c}
2 k \\
k
\end{array}\right)\left(\frac{c^{2}-4 d}{16(2 c)^{2}}\right)^{k} \\
\equiv & \left(\frac{-1}{p}\right) \sum_{j=0}^{p-1}\left(\frac{j}{p}\right)\left(\frac{i^{2}+2 c i j+\left(c^{2}-4 d\right) j^{2}}{p}\right)(\bmod p) .
\end{aligned}
$$

Thus $D \equiv 0(\bmod p)$. Clearly $|D|<2 p$.

If $p \mid i$, then

$$
\begin{aligned}
D & =\sum_{j=0}^{p-1}\left(\frac{j}{p}\right)\left(\frac{d j^{2}}{p}\right)-\sum_{j=0}^{p-1}\left(\frac{-j}{p}\right)\left(\frac{\left(c^{2}-4 d\right) j^{2}}{p}\right) \\
& =\left(\left(\frac{d}{p}\right)-\left(\frac{4 d-c^{2}}{p}\right)\right) \sum_{j=1}^{p-1}\left(\frac{j}{p}\right)=0 .
\end{aligned}
$$

Now assume that $p \nmid i$. If neither $c^{2}-4 d$ nor $(2 c)^{2}-4\left(c^{2}-4 d\right)=16 d$ is divisible by $p$, then

$$
\left|\left\{1 \leqslant j \leqslant p-1: i^{2}+c i j+d j^{2} \equiv 0(\bmod p)\right\}\right| \in\{0,2\}
$$

and

$$
\left|\left\{1 \leqslant j \leqslant p-1: i^{2}+2 c i j+\left(c^{2}-4 d\right) j^{2} \equiv 0(\bmod p)\right\}\right| \in\{0,2\},
$$

hence $D$ is even. When $p \mid d$, we also have $2 \mid D$ since

$$
|\{1 \leqslant j \leqslant p-1: p \mid i(i+c j)\}|=\left|\left\{1 \leqslant j \leqslant p-1: p \mid i(i+c j)^{2}\right\}\right|=1 .
$$


If $p \mid c^{2}-4 d$, then $2 \mid D$ since

$$
|\{1 \leqslant j \leqslant p-1: p \mid i(i+2 c j)\}|=1
$$

and

$$
\left|\left\{1 \leqslant j \leqslant p-1: i^{2}+c i j+d j^{2} \equiv\left(i+\frac{c}{2} j\right)^{2} \equiv 0(\bmod p)\right\}\right|=1 .
$$

So $D$ is always even, and hence $D=0$ as $p \mid D$ and $|D|<2 p$.

Case 2. $n=p_{1} \ldots p_{r}$ with $r \geqslant 2$, where $p_{1}, \ldots, p_{r}$ are distinct primes.

By the Chinese Remainder Theorem,

$$
\begin{aligned}
\sum_{j=0}^{n-1}\left(\frac{j}{n}\right)\left(\frac{i^{2}+c i j+d j^{2}}{n}\right) & =\sum_{j=0}^{n-1} \prod_{s=1}^{r}\left(\frac{j}{p_{s}}\right)\left(\frac{i^{2}+c i j+d j^{2}}{p_{s}}\right) \\
& =\sum_{j_{1}=0}^{p_{1}-1} \ldots \sum_{j_{r}=0}^{p_{r}-1} \prod_{s=1}^{r}\left(\frac{j_{s}}{p_{s}}\right)\left(\frac{i^{2}+c i j_{s}+d j_{s}^{2}}{p_{s}}\right)
\end{aligned}
$$

and hence

$$
\sum_{j=0}^{n-1}\left(\frac{j}{n}\right)\left(\frac{i^{2}+c i j+d j^{2}}{n}\right)=\prod_{s=1}^{r} \sum_{j_{s}=0}^{p_{s}-1}\left(\frac{j_{s}}{p_{s}}\right)\left(\frac{i^{2}+c i j_{s}+d j_{s}^{2}}{p_{s}}\right) .
$$

Similarly,

$$
\begin{aligned}
& \sum_{j=0}^{n-1}\left(\frac{-j}{n}\right)\left(\frac{i^{2}+2 c i j+\left(c^{2}-4 d\right) j^{2}}{n}\right) \\
= & \prod_{s=1}^{r} \sum_{j_{s}=0}^{p_{s}-1}\left(\frac{-j_{s}}{p_{s}}\right)\left(\frac{i^{2}+2 c i j_{s}+\left(c^{2}-4 d\right) j_{s}^{2}}{p_{s}}\right) .
\end{aligned}
$$

Thus, (1.5) holds in view of Case 1. This concludes the proof.

\section{Proofs of Theorems 1.3 And 1.1}

Lemma 3.1. Let $p>3$ be a prime. If $p \equiv 1,3(\bmod 8)$ and $p=x^{2}+2 y^{2}$ with $x, y \in \mathbb{Z}$ and $x \equiv 1(\bmod 4)$, then

$$
\sum_{k=0}^{p-1} \frac{\left(\begin{array}{c}
4 k \\
2 k
\end{array}\right)\left(\begin{array}{c}
2 k \\
k
\end{array}\right)}{128^{k}} \equiv(-1)^{\lfloor(p+5) / 8\rfloor}\left(2 x-\frac{p}{2 x}\right)\left(\bmod p^{2}\right) .
$$

If $\left(\frac{-2}{p}\right)=-1$, i.e., $p \equiv 5,7(\bmod 8)$, then

$$
\sum_{k=0}^{p-1} \frac{\left(\begin{array}{c}
4 k \\
2 k
\end{array}\right)\left(\begin{array}{c}
2 k \\
k
\end{array}\right)}{128^{k}} \equiv 0\left(\bmod p^{2}\right) \text {. }
$$


Remark 3.1. The first assertion in Lemma 3.1 was conjectured by Z.-W. Sun [8] and confirmed by his twin brother Z.-H. Sun [7, Theorem 4.3]. The second assertion was proved by Z.-W. Sun [9, Corollary 1.3] as a consequence of (2.3) with $x=1 / 2$.

Lemma 3.2. Let $p$ be an odd prime and let $c, d, i \in \mathbb{Z}$ with $p \nmid d$. Then

$$
\sum_{j=0}^{p-1}\left(\frac{j}{p}\right)\left(\frac{i^{2}+3 c i j+d j^{2}}{p}\right)=\left(\frac{i}{p}\right) \sum_{x=0}^{p-1}\left(\frac{x^{3}-\left(3 c^{2}-d\right) x+c\left(2 c^{2}-d\right)}{p}\right) .
$$

Proof. Both sides of (3.1) vanish if $p \mid i$. Below we assume $p \nmid i$.

Clearly,

$$
\begin{aligned}
& \sum_{j=0}^{p-1}\left(\frac{j}{p}\right)\left(\frac{i^{2}+3 c i j+d j^{2}}{p}\right) \\
= & \sum_{j=0}^{p-1}\left(\frac{d j}{p}\right)\left(\frac{d i^{2}+3 c i(d j)+(d j)^{2}}{p}\right) \\
= & \sum_{k=0}^{p-1}\left(\frac{k}{p}\right)\left(\frac{k^{2}+3 c i k+d i^{2}}{p}\right)=\sum_{r=0}^{p-1}\left(\frac{i r}{p}\right)\left(\frac{(i r)^{2}+3 c i^{2} r+d i^{2}}{p}\right) \\
= & \left(\frac{i}{p}\right) \sum_{r=0}^{p-1}\left(\frac{r}{p}\right)\left(\frac{r^{2}+3 c r+d}{p}\right)
\end{aligned}
$$

and

$$
\begin{aligned}
\sum_{r=0}^{p-1}\left(\frac{r^{3}+3 c r^{2}+d r}{p}\right) & =\sum_{x=0}^{p-1}\left(\frac{(x-c)^{3}+3 c(x-c)^{2}+d(x-c)}{p}\right) \\
& =\sum_{x=0}^{p-1}\left(\frac{x^{3}+\left(d-3 c^{2}\right) x+c\left(2 c^{2}-d\right)}{p}\right) .
\end{aligned}
$$

So (3.1) holds.

Lemma 3.3. Let $p$ be any odd prime and let $i \in \mathbb{Z}$.

(i) We have

$$
\begin{aligned}
& \sum_{j=0}^{p-1}\left(\frac{j}{p}\right)\left(\frac{i^{2}+4 i j+2 j^{2}}{p}\right) \\
= & \begin{cases}(-1)^{\lfloor(p-3) / 8\rfloor}\left(\frac{i}{p}\right) 2 x & \text { if } p=x^{2}+2 y^{2}(x, y \in \mathbb{Z} \& 4 \mid x-1), \\
0 & \text { if }\left(\frac{-2}{p}\right)=-1, \text { i.e., } p \equiv 5,7(\bmod 8) .\end{cases}
\end{aligned}
$$


(ii) We have

$$
\begin{aligned}
& \sum_{j=0}^{p-1}\left(\frac{j}{p}\right)\left(\frac{i^{2}+3 i j+2 j^{2}}{p}\right) \\
= & \begin{cases}-\left(\frac{2 i}{p}\right) 2 x & \text { if } p=x^{2}+4 y^{2}(x, y \in \mathbb{Z} \& 4 \mid x-1), \\
0 & \text { if }\left(\frac{-1}{p}\right)=-1, \text { i.e., } p \equiv 3(\bmod 4) .\end{cases}
\end{aligned}
$$

Also,

$$
\begin{aligned}
& \sum_{j=0}^{p-1}\left(\frac{j}{p}\right)\left(\frac{i^{2}+3 i j+3 j^{2}}{p}\right) \\
= & \begin{cases}-\left(\frac{-i}{p}\right) 2 x & \text { if } p=x^{2}+3 y^{2}(x, y \in \mathbb{Z} \& 3 \mid x-1), \\
0 & \text { if }\left(\frac{-3}{p}\right) \neq 1, \text { i.e., } p \equiv 0,2(\bmod 3),\end{cases}
\end{aligned}
$$

and

$$
\begin{aligned}
& \sum_{j=0}^{p-1}\left(\frac{j}{p}\right)\left(\frac{i^{2}+21 i j+112 j^{2}}{p}\right) \\
= & \begin{cases}-\left(\frac{i}{p}\right) 2 x & \text { if } p=x^{2}+7 y^{2}\left(x, y \in \mathbb{Z} \&\left(\frac{x}{7}\right)=1\right), \\
0 & \text { if }\left(\frac{-7}{p}\right) \neq 1, \text { i.e., } p \equiv 0,3,5,6(\bmod 7) .\end{cases}
\end{aligned}
$$

Remark 3.2. It is well known that any prime $p \equiv 1(\bmod 4)$ can be written as $x^{2}+4 y^{2}$ with $x, y \in \mathbb{Z}$. Also, for each $m \in\{2,3,7\}$ any odd prime $p$ with $\left(\frac{-m}{p}\right)=1$ can be written $x^{2}+m y^{2}$ with $x, y \in \mathbb{Z}$ (cf. [3]).

Proof of Lemma 3.3. It is easy to verify that (3.2)-(3.5) hold for $p=3$. Below we assume $p>3$.

(i) As $16 \times 4^{2} / 2=128$, combining Lemma 2.1 and Lemma 3.1 we find that

$$
\begin{aligned}
& \sum_{j=0}^{p-1}\left(\frac{j}{p}\right)\left(\frac{i^{2}+4 i j+2 j^{2}}{p}\right) \\
\equiv & \begin{cases}(-1)^{\lfloor(p-3) / 8\rfloor}\left(\frac{i}{p}\right) 2 x(\bmod p) & \text { if } p=x^{2}+2 y^{2}(x, y \in \mathbb{Z} \& 4 \mid x-1), \\
0(\bmod p) & \text { if }\left(\frac{-2}{p}\right)=-1, \text { i.e., } p \equiv 5,7(\bmod 8) .\end{cases}
\end{aligned}
$$

Observe that

$$
\sum_{j=0}^{p-1}\left(\frac{j}{p}\right)\left(\frac{i^{2}+4 i j+2 j^{2}}{p}\right)=\sum_{j=1}^{p-1}\left(\frac{j}{p}\right)\left(\frac{i^{2}+4 i j+2 j^{2}}{p}\right)
$$

is even $\left(\right.$ since $\left.\left|\left\{1 \leqslant j \leqslant p-1: i^{2}+4 i j+2 j^{2} \equiv 0(\bmod p)\right\}\right| \in\{0,2\}\right)$, and its absolute value is smaller than $p$. If $p=x^{2}+2 y^{2}$ with $x, y \in \mathbb{Z}$ and $x \equiv 1(\bmod 4)$, then $|2 x|<2 \sqrt{p}<p$. So $(3.2)$ holds. 
(ii) In light of Lemma 3.2,

$$
\begin{aligned}
& \sum_{j=0}^{p-1}\left(\frac{j}{p}\right)\left(\frac{i^{2}+3 i j+2 j^{2}}{p}\right) \\
= & \left(\frac{i}{p}\right) \sum_{r=0}^{p-1}\left(\frac{r^{3}-r}{p}\right)=\left(\frac{2 i}{p}\right) \sum_{r=0}^{p-1}\left(\frac{2 r}{p}\right)\left(\frac{4 r^{2}-4}{p}\right) \\
= & \left(\frac{2 i}{p}\right) \sum_{s=0}^{p-1}\left(\frac{s^{3}-4 s}{p}\right)
\end{aligned}
$$

and

$$
\begin{aligned}
& \sum_{j=0}^{p-1}\left(\frac{j}{p}\right)\left(\frac{i^{2}+3 i j+3 j^{2}}{p}\right) \\
= & \left(\frac{i}{p}\right) \sum_{r=0}^{p-1}\left(\frac{r^{3}-1}{p}\right)=\left(\frac{2 i}{p}\right) \sum_{r=0}^{p-1}\left(\frac{(2 r)^{3}-8}{p}\right) \\
= & \left(\frac{2 i}{p}\right) \sum_{s=0}^{p-1}\left(\frac{s^{3}-8}{p}\right) .
\end{aligned}
$$

On the other hand, by [1, Theorem 6.2.9] and [1, pp. 195-196],

$$
\sum_{s=0}^{p-1}\left(\frac{s^{3}-4 s}{p}\right)= \begin{cases}-2 x & \text { if } p=x^{2}+4 y^{2}(x, y \in \mathbb{Z} \& 4 \mid x-1), \\ 0 & \text { if } p \equiv 3(\bmod 4),\end{cases}
$$

and

$$
\sum_{s=0}^{p-1}\left(\frac{s^{3}-8}{p}\right)= \begin{cases}-2 x\left(\frac{-2}{p}\right) & \text { if } p=x^{2}+3 y^{2}(x, y \in \mathbb{Z} \& 3 \mid x-1), \\ 0 & \text { if } p \equiv 2(\bmod 3) .\end{cases}
$$

So we have (3.3) and (3.4).

Now we prove (3.5). Clearly, (3.5) is valid if $p \mid i$ or $p=7$. Below we assume that $p \nmid i$ and $p \neq 7$. Observe that

$$
\begin{aligned}
& \sum_{j=0}^{p-1}\left(\frac{j}{p}\right)\left(\frac{i^{2}+21 i j+112 j^{2}}{p}\right) \\
= & \sum_{r=0}^{p-1}\left(\frac{112 i r}{p}\right)\left(\frac{112 i^{2}+21 i^{2}(112 r)+(112 i r)^{2}}{p}\right) \\
= & \left(\frac{i}{p}\right) \sum_{s=0}^{p-1}\left(\frac{s^{3}+21 s^{2}+112 s}{p}\right) .
\end{aligned}
$$


By a result of Rajwade [6],

$$
\sum_{s=0}^{p-1}\left(\frac{s^{3}+21 s^{2}+112 s}{p}\right)=\left\{\begin{array}{ll}
-2 x & \text { if } p=x^{2}+7 y^{2} \\
0 & \text { if }\left(\frac{-7}{p}\right)=-1 .
\end{array}\left(x, y \in \mathbb{Z} \&\left(\frac{x}{7}\right)=1\right),\right.
$$

Therefore (3.5) holds.

The proof of Lemma 3.3 is now complete.

Proof of Theorem 1.3. Write $n=p_{1} \ldots p_{r}$ with $p_{1}, \ldots, p_{r}$ distinct primes. In light of (2.4) and Lemma 3.3(i), if $-2 R n$ fails (i.e., $\left(\frac{-2}{p_{s}}\right)=-1$ for some $s=1, \ldots, r)$ then

$$
\sum_{j=0}^{n-1}\left(\frac{j}{n}\right)\left(\frac{i^{2}+4 i j+2 j^{2}}{n}\right)=\prod_{s=1}^{r} \sum_{j=0}^{p_{s}-1}\left(\frac{j_{s}}{p_{s}}\right)\left(\frac{i^{2}+4 i j_{s}+2 j_{s}^{2}}{p_{s}}\right)=0,
$$

Thus (1.7) holds. Note that if $-2 R n$ then for each $s=1, \ldots, r$ we may write $p_{s}=x_{s}^{2}+2 y_{s}^{2}$ with $x_{s}, y_{s} \in \mathbb{Z}$ and $x_{s} \equiv 1(\bmod 4)$ and hence

$$
\left.\sum_{j=0}^{n-1}\left(\frac{j}{n}\right)\left(\frac{i^{2}+4 i j+2 j^{2}}{n}\right)=\prod_{s=1}^{r}(-1)^{\left\lfloor\left(p_{s}-3\right) / 8\right\rfloor}\left(\frac{i}{p_{s}}\right) 2 x_{s}\right) .
$$

Similarly, (1.6), (1.8) and (1.9) also hold in view of (2.4) and Lemma 3.3(ii). This concludes our proof of Theorem 1.3.

Proof of Theorem 1.1. Suppose that $n=\prod_{s=1}^{r} p_{s}^{a_{s}}$, where $p_{1}, \ldots, p_{r}$ are distinct primes and $a_{1}, \ldots, a_{r}$ are positive integers. If $a_{t}>1$ with $1 \leqslant t \leqslant r$, then $n / p_{t} \equiv 0\left(\bmod p_{1} \ldots p_{r}\right)$ and hence for any $i \in \mathbb{Z}$ we have

$$
\begin{aligned}
\left(\frac{i^{2}+c i j+d j^{2}}{n}\right) & =\prod_{s=1}^{r}\left(\frac{i^{2}+c i j+d j^{2}}{p_{s}}\right)^{a_{s}} \\
& =\prod_{s=1}^{r}\left(\frac{\left(i+n / p_{t}\right)^{2}+c\left(i+n / p_{t}\right) j+d j^{2}}{p_{s}}\right)^{a_{s}} \\
& =\left(\frac{\left(i+n / p_{t}\right)^{2}+c\left(i+n / p_{t}\right) j+d j^{2}}{n}\right)
\end{aligned}
$$

for all $j=0, \ldots, n-1$. Therefore

$$
(c, d)_{n}=[c, d]_{n}=0 .
$$

Below we assume that $n$ is squarefree. If $-1 R n$ fails, then by Theorems 1.2 and 1.3 we have

$$
\sum_{j=1}^{n-1}\left(\frac{j}{n}\right)\left(\frac{i^{2}+3 i j+2 j^{2}}{n}\right)=0=\sum_{j=1}^{n-1}\left(\frac{j}{n}\right)\left(\frac{i^{2}+6 i j+j^{2}}{n}\right)
$$

for all $i=0, \ldots, n-1$, hence $(3,2)_{n}=(6,1)_{n}=0$ and $[3,2]_{n}=[6,1]_{n}=0$. This proves part (i) of Theorem 1.1. Similarly, parts (ii)-(iv) of Theorem 1.1 follow from Theorems 1.2 and 1.3. This ends the proof. 


\section{Proof of Theorem 1.4}

Let $q>1$ be a prime power and let $\mathbb{F}_{q}$ be the finite field of order $q$. A multiplicative character $\chi$ on $\mathbb{F}_{q}$ is called trivial (or principal) if $\chi(a)=1$ for all $a \in \mathbb{F}_{q}^{*}=\mathbb{F}_{q} \backslash\{0\}$. For a polynomial $P(x)=\sum_{s=0}^{n} c_{s} x^{s} \in \mathbb{F}_{q}[x]$, we define the homogenous polynomial

$$
P^{*}(x, y)=\sum_{s=0}^{n} c_{s} x^{n-s} y^{s}=x^{n} P\left(\frac{y}{x}\right) .
$$

Fix a list of the elements of $\mathbb{F}_{q}$. For a multiplicative character $\chi$ on $\mathbb{F}_{q}$, we introduce the matrices

$$
M(P, \chi)=\left[\chi\left(P^{*}(a, b)\right)\right]_{a, b \in \mathbb{F}_{q}^{*}} \text { and } M_{0}(P, \chi)=\left[\chi\left(P^{*}(a, b)\right)\right]_{a, b \in \mathbb{F}_{q}} .
$$

Lemma 4.1. Let $q>1$ be a prime power and let $\chi$ be a nontrivial multiplicative character on $\mathbb{F}_{q}$. Suppose that $P(x) \in \mathbb{F}_{q}[x]$ and $\sum_{x \in \mathbb{F}_{q}} \chi(x P(x))=0$. Then $M(P, \chi)$ is singular (i.e., det $M(P, \chi)=0)$. If the character $\chi^{n+1}$ is nontrivial with $n=\operatorname{deg} P$, then the matrix $M_{0}(P, \chi)$ is singular too.

Proof. We introduce the column vector $v$ whose coordinates are $v_{b}=\chi(b)$ for $b \in \mathbb{F}_{q}^{*}$. Let $M=M(P, \chi)$. Then, for any $a \in \mathbb{F}_{q}^{*}$ we have

$$
(M v)_{a}=\sum_{b \in \mathbb{F}_{q}^{*}} \chi\left(a^{n} P\left(a^{-1} b\right)\right) \chi(b)=\chi\left(a^{n+1}\right) \sum_{b \in \mathbb{F}_{q}^{*}} \chi\left(a^{-1} b P\left(a^{-1} b\right)\right)=0 .
$$

Since $v$ is a nonzero vector, the matrix $M$ is singular.

Now suppose that the degree of $P$ is $n$ and the character $\chi^{n+1}$ is nontrivial. Let $M_{0}=M_{0}(P, \chi)$ and introduce the vector $v$ with coordinates $v_{b}=\chi(b)$ for $b \in \mathbb{F}_{q}$. Then $\left(M_{0} v\right)_{a}=0$ for all $a \in \mathbb{F}_{q}^{*}$ as before. Let $c_{n}$ be the leading coefficient of the polynomial $P(x)$. Then

$$
\left(M_{0} v\right)_{0}=\sum_{b \in \mathbb{F}_{q}} \chi\left(c_{n} b^{n}\right) \chi(b)=\chi\left(c_{n}\right) \sum_{b \in \mathbb{F}_{q}} \chi^{n+1}(b)=0 .
$$

Therefore $M_{0} v$ is the zero vector and hence $M_{0}$ is singular.

Motivated by Lemma 4.1, we give the following more sophisticated lemma.

Lemma 4.2. Let $q>1$ be an odd prime power. Suppose that $g \in \mathbb{F}_{q}$ is not a square and $\chi$ is a nontrivial multiplicative character on $\mathbb{F}_{q}$ with $\chi(-1)=1$. Assume that $P(x) \in \mathbb{F}_{q}[x]$ and

$$
\sum_{x \in \mathbb{F}_{q}} \chi\left(x P\left(x^{2}\right)\right)=\sum_{x \in \mathbb{F}_{q}} \chi\left(x P\left(g x^{2}\right)\right)=0 .
$$

(i) We have $\operatorname{dim}(\operatorname{Ker}(M(P, \chi))) \geqslant 2$, in particular $M(P, \chi)$ is singular.

(ii) Assume that the character $\chi^{2 n+1}$ with $n=\operatorname{deg} P$ is nontrivial. Then $\operatorname{dim}\left(\operatorname{Ker}\left(M_{0}(P, \chi)\right)\right) \geqslant 2$. 
Proof. For $a, b \in \mathbb{F}_{q}$, set

$$
v_{a, b}:= \begin{cases}\chi(c)=\chi(\sqrt{a b}) & \text { if } a b=c^{2} \text { for some } c \in \mathbb{F}_{q}, \\ 0 & \text { otherwise. }\end{cases}
$$

This is well defined since $\chi( \pm 1)=1$, The matrix $V=\left[v_{a, b}\right]_{a, b \in \mathbb{F}_{q}^{*}}$ has rank 2 ; in fact, if $b^{\prime}=b c^{2}$ for some $c \in \mathbb{F}_{q}$ then columns $b$ and $b^{\prime}$ in $V$ are proportional, but columns 1 and $g$ are not proportional.

(i) Write $M$ for $M(P, \chi)$. It suffices to show that $M V$ is the zero matrix. For $a, b \in \mathbb{F}_{q}^{*}$, the $(a, b)$-entry of the matric $M V$ is

$$
\begin{aligned}
\sum_{c \in \mathbb{F}_{q}} \chi\left(P^{*}(a, c)\right) v_{c, b} & =\sum_{\substack{c \in \mathbb{F}_{q} \\
b c \text { is a square }}} \chi\left(a^{n} P\left(a^{-1} c\right)\right) \chi(\sqrt{b c}) \\
& =\frac{1}{2} \sum_{d \in \mathbb{F}_{q}} \chi\left(a^{n} P\left(a^{-1} b d^{2}\right)\right) \chi(b d) \\
& =\frac{1}{2} \chi\left(a^{n} b\right) \sum_{d \in \mathbb{F}_{q}} \chi\left(P_{a^{-1} b}(d)\right)
\end{aligned}
$$

where $P_{c}(x)=x P\left(c x^{2}\right)$ for any $c \in \mathbb{F}_{q}$.

Now it remains to show for any $c \in \mathbb{F}_{q}^{*}$ the identity

$$
\sum_{x \in \mathbb{F}_{q}} \chi\left(P_{c}(x)\right)=0
$$

Clearly, $c=c_{0} d^{2}$ for some $c_{0} \in\{1, g\}$ and $d \in \mathbb{F}_{q}^{*}$. Thus

$$
\begin{aligned}
\sum_{x \in \mathbb{F}_{q}} \chi\left(P_{c}(x)\right) & =\sum_{x \in \mathbb{F}_{q}} \chi\left(x P\left(c_{0} d^{2} x^{2}\right)\right)=\sum_{y \in \mathbb{F}_{q}} \chi\left(d^{-1} y P\left(c_{0} y^{2}\right)\right) \\
& =\chi(d)^{-1} \sum_{y \in \mathbb{F}_{q}} \chi\left(P_{c_{0}}(y)\right)=0 .
\end{aligned}
$$

This proves part (i) of Lemma 4.2.

(ii) Write $M_{0}$ for $M_{0}(P, \chi)$, and define $V_{0}=\left[v_{a, b}\right]_{a, b \in \mathbb{F}_{q}}$. (Note the slight difference between $V_{0}$ and $V$.) The rank of $V_{0}$ is still equal to 2 , so it suffices to show that $M_{0} V_{0}$ is the zero matrix. Note that the $(a, b)$-entry of $M_{0} V_{0}$ is trivially zero if $b=0$ since $v_{c, 0}=0$ for all $c \in \mathbb{F}_{q}$. For $a, b \neq 0$ we can repeat the computation for $M V$ verbatim. Let $c_{n}$ denote the leading coefficient of $P(x)$. If $a=0$ and $b \neq 0$, then the $(a, b)$-entry of $M_{0} V_{0}$ is

$$
\begin{aligned}
\sum_{c \in \mathbb{F}_{q}} \chi\left(P^{*}(0, c)\right) v_{c, b}= & \sum_{\substack{c \in \mathbb{F}_{q} \\
b c \text { is a square }}} \chi\left(c_{n} c^{n}\right) \chi(\sqrt{b c}) \\
& =\frac{1}{2} \sum_{d \in \mathbb{F}_{q}^{*}} \chi\left(c_{n}\left(b^{-1} d^{2}\right)^{n} d\right)=\frac{\chi\left(b^{-1} c_{n}\right)}{2} \sum_{d \in \mathbb{F}_{q}} \chi^{2 n+1}(d) .
\end{aligned}
$$

This is zero since $\chi^{2 n+1}$ is nontrivial. We are done. 
Theorem 4.1. Let $q>1$ be an odd prime power and let $m \in \mathbb{Z}^{+}$with $\operatorname{gcd}(m, q-1)=1$. Let $\chi$ be a nontrivial quadratic character on $\mathbb{F}_{q}$, and let

$$
P_{m}(x, a)=\sum_{k=0}^{m-1}\left(\begin{array}{c}
2 m \\
2 k+1
\end{array}\right) a^{k} x^{m-1-k}
$$

with $a \in \mathbb{F}_{q}^{*}=\mathbb{F}_{q} \backslash\{0\}$. Then

$$
\sum_{x \in \mathbb{F}_{q}} \chi\left(x P_{m}\left(g x^{2}, a\right)\right)=0 \quad \text { for all } g \in \mathbb{F}_{q}^{*} .
$$

If $\chi(-1)=1$, then both $M\left(P_{m}(x, a), \chi\right)$ and $M_{0}\left(P_{m}(x, a), \chi\right)$ are singular, and moreover either of them has a kernel of dimension at least two.

Proof. In view of Lemma 4.2, we only need to prove (4.5). As $P_{m}\left(g x^{2}, a\right)=$ $g^{m-1} P_{m}\left(x^{2}, a g^{-1}\right)$ for all $g \in \mathbb{F}_{q}^{*}$, it suffices to show that

$$
\sum_{x \in \mathbb{F}_{q}} \chi\left(x P_{m}\left(x^{2}, a\right)\right)=0
$$

for any $a \in \mathbb{F}_{q}^{*}$.

Clearly, $m$ is odd since $\operatorname{gcd}(m, q-1)=1$. Recall that $\chi^{2}$ is the trivial character, and note that

$$
\begin{aligned}
\sum_{x \in \mathbb{F}_{q}} \chi\left(x P_{m}\left(x^{2}, a\right)\right) & =\sum_{x \in \mathbb{F}_{q}^{*}} \chi\left(a x^{-1} P_{m}\left(\left(a x^{-1}\right)^{2}, a\right)\right) \\
& =\sum_{x \in \mathbb{F}_{q}^{*}} \chi\left(\sum_{k=0}^{m-1}\left(\begin{array}{c}
2 m \\
2(m-1-k)+1
\end{array}\right) a^{2 m-1-k} x^{2 k+1-2 m}\right) \\
& =\sum_{x \in \mathbb{F}_{q}^{*}} \chi\left(\sum_{j=0}^{m-1}\left(\begin{array}{c}
2 m \\
2 j+1
\end{array}\right) a^{m+j} x^{-1-2 j}\right) \\
& =\sum_{x \in \mathbb{F}_{q}^{*}} \chi\left(a^{m} x^{-2 m} x P_{m}\left(x^{2}, a\right)\right)=\chi(a)^{m} \sum_{x \in \mathbb{F}_{q}} \chi\left(x P_{m}\left(x^{2}, a\right)\right) .
\end{aligned}
$$

If $a$ is not a square in $\mathbb{F}_{q}$, then $\chi(a)^{m}=(-1)^{m}=-1$ and hence (4.6) holds by the above.

Now assume that $a=b^{2}$ with $b \in \mathbb{F}_{q}^{*}$. Since

$$
\sum_{x \in \mathbb{F}_{q}} \chi\left(x P_{m}\left(x^{2}, a\right)\right)=\sum_{x \in \mathbb{F}_{q}} \chi\left(b^{2 m-2} x P_{m}\left(\left(b^{-1} x\right)^{2}, 1\right)\right)=\chi(b)^{2 m-1} \sum_{y \in \mathbb{F}_{q}} \chi\left(y P_{m}\left(y^{2}, 1\right)\right),
$$

it remains to show that $\sum_{x \in \mathbb{F}_{q}} \chi\left(x P_{m}\left(x^{2}, 1\right)\right)=0$. Since $\chi=\chi^{-1}$ and $2 x P_{m}\left(x^{2}, 1\right)=(x+1)^{2 m}-(x-1)^{2 m}=\left((x+1)^{m}+(x-1)^{m}\right)\left((x+1)^{m}-(x-1)^{m}\right)$, we have

$$
\sum_{x \in \mathbb{F}_{q}} \chi\left(2 x P_{m}\left(x^{2}, 1\right)\right)
$$




$$
\begin{aligned}
& =\chi\left(2^{2 m}\right)+\sum_{x \in \mathbb{F}_{q} \backslash\{1\}} \chi\left((x+1)^{m}+(x-1)^{m}\right) \chi^{-1}\left((x+1)^{m}-(x-1)^{m}\right) \\
& =1+\sum_{x \in \mathbb{F}_{q} \backslash\{1\}} \chi\left(\frac{(x+1)^{m}+(x-1)^{m}}{(x+1)^{m}-(x-1)^{m}}\right) \\
& =1+\sum_{x \in \mathbb{F}_{q} \backslash\{1\}} \chi\left(\frac{(1+2 /(x-1))^{m}+1}{(1+2 /(x-1))^{m}-1}\right) \\
& =1+\sum_{y \in \mathbb{F}_{q} \backslash\{1\}} \chi\left(\frac{y^{m}+1}{y^{m}-1}\right)=1+\sum_{y \in \mathbb{F}_{q} \backslash\{1\}} \chi\left(\frac{y+1}{y-1}\right) \\
& =1+\sum_{y \in \mathbb{F}_{q} \backslash\{1\}} \chi\left(1+\frac{2}{y-1}\right)=1+\sum_{z \in \mathbb{F}_{q} \backslash\{1\}} \chi(z)=0
\end{aligned}
$$

Thus $\sum_{x \in \mathbb{F}_{q}} \chi\left(x P_{m}\left(x^{2}, 1\right)\right)=0$ as desired.

The proof of Theorem 4.1 is now complete.

Proof of Theorem 1.4(i). Let $p$ be any prime with $p \equiv 5(\bmod 12)$, and let $\chi$ be the quadratic character of $\mathbb{F}_{p}=\mathbb{Z} / p \mathbb{Z}$ with $\chi(x+p \mathbb{Z})=\left(\frac{x}{p}\right)$ for all $x \in \mathbb{Z}$. Note that $\chi(-1)=1$ since $p \equiv 1(\bmod 4)$. Clearly,

$$
P_{3}(x, 3)=\left(\begin{array}{l}
6 \\
1
\end{array}\right) x^{2}+\left(\begin{array}{l}
6 \\
3
\end{array}\right) 3 x+\left(\begin{array}{l}
6 \\
5
\end{array}\right) 3^{2}=6\left(x^{2}+10 x+9\right) .
$$

Applying Theorem 4.1, we obtain that

$$
(10,9)_{p}=\operatorname{det}\left[\left(\frac{i^{2}+10 i j+9 j^{2}}{p}\right)\right]_{1 \leqslant i, j \leqslant p-1}=0
$$

and

$$
[10,9]_{p}=\operatorname{det}\left[\left(\frac{i^{2}+10 i j+9 j^{2}}{p}\right)\right]_{0 \leqslant i, j \leqslant p-1}=0 .
$$

Note that Sun stated in [S19, Remark 4.9] that $(10,9)_{p}=0$ if and only if $[10,9]_{p}=0$.

Let $\mathbb{F}_{q}$ be a finite field of order $q$. A polynomial $P(x) \in \mathbb{F}_{q}[x]$ is called a permutation polynomial if $P$ is bijective as a function on $\mathbb{F}_{q}$. If $\chi$ is a nontrivial multiplicative character on $\mathbb{F}_{q}$ and $P(x) \in \mathbb{F}_{q}[x]$ is a permutation polynomial, then

$$
\sum_{x \in \mathbb{F}_{q}} \chi(P(x))=\sum_{y \in \mathbb{F}_{q}} \chi(y)=0
$$

and also

$$
\sum_{x \in \mathbb{F}_{q}^{*}} \chi(P(x))=0
$$

provided that $P(0)=0$. 
Theorem 4.2. Let $q>1$ be an odd prime power and let $m \in \mathbb{Z}^{+}$with $\operatorname{gcd}\left(m, q^{2}-1\right)=1$. Let $\chi$ be a nontrivial multiplicative character on $\mathbb{F}_{q}$ with $\chi(-1)=1$. For the polynomial

$$
Q_{m}(x, a):=\sum_{i=0}^{(m-1) / 2} \frac{m}{m-i}\left(\begin{array}{c}
m-i \\
i
\end{array}\right)(-a)^{i} x^{(m-1) / 2-i}
$$

with $a \in \mathbb{F}_{q}^{*}$, we have

$$
\operatorname{dim}\left(\operatorname{Ker}\left(M\left(Q_{m}(x, a), \chi\right)\right)\right) \geqslant 2 .
$$

Moreover, if the character $\chi^{m}$ is nontrivial, then

$$
\operatorname{dim}\left(\operatorname{Ker}\left(M_{0}\left(Q_{m}(x, a), \chi\right)\right)\right) \geqslant 2 .
$$

Proof. Let $a \in \mathbb{F}_{q}$. It is a classical result (cf. [5, pp. 355-357]) that the Dickson polynomial $D_{m}(x, a):=x Q_{m}\left(x^{2}, a\right)$ is a permutation polynomial on $\mathbb{F}_{q}$. For any $g \in \mathbb{F}_{q}^{*}$, as $Q_{m}\left(g x^{2}, a\right)=g^{(m-1) / 2} Q_{m}\left(x^{2}, a g^{-1}\right)$, the polynomial $x Q_{m}\left(g x^{2}, a\right)$ is also a permutation polynomial on $\mathbb{F}_{q}$. Thus

$$
\sum_{x \in \mathbb{F}_{q}} \chi\left(x Q_{m}\left(g x^{2}, a\right)\right)=0 \quad \text { for all } g \in \mathbb{F}_{q}^{*} .
$$

Combining this with Lemma 4.2, we immediately obtain the desired results.

Proof of Theorem 1.4(ii). Let $p$ be any prime with $p \equiv 13,17(\bmod 20)$. Then $\operatorname{gcd}\left(5, p^{2}-1\right)=1$. Let $\chi$ be the quadratic character of $\mathbb{F}_{p}=\mathbb{Z} / p \mathbb{Z}$ with $\chi(x+p \mathbb{Z})=\left(\frac{x}{p}\right)$ for all $x \in \mathbb{Z}$. Then $\chi(-1)=1$ since $p \equiv 1(\bmod 4)$. Clearly $\chi^{5}=\chi$ is nontrivial and $Q_{5}(x,-1)=x^{2}+5 x+5$. Applying Theorem 4.2 , we get that

$$
[5,5]_{p}=\operatorname{det}\left[\left(\frac{i^{2}+5 i j+5 j^{2}}{p}\right)\right]_{0 \leqslant i, j \leqslant p-1}=0 .
$$

This concludes the proof.

Note that actually our method to prove Theorem 1.4 yields a stronger result stated after Theorem 1.4 in Section 1.

\section{A SUfFicient CONDition FOR $\sum_{x=0}^{p-1}\left(\frac{a x^{5}+b x^{3}+c x}{p}\right)=0$}

For an odd prime power $q>1$, we let $\chi_{q}$ denote the quadratic multiplicative character on the finite field $\mathbb{F}_{q}$.

Let $p \equiv 1(\bmod 4)$ be a prime and let $a$ be a nonzero element of $\mathbb{F}_{p}$. If $\chi_{p}(a)=1$, then we define $\sqrt{a}$ as an element $\alpha \in \mathbb{F}_{p}$ with $\alpha^{2}=a$. When $\chi_{p}(a)=-1$, the finite field $\mathbb{F}_{p^{2}} \cong \mathbb{F}_{p}[x] /\left(x^{2}-a\right)$ contains an element $\alpha$ with $\alpha^{2}=a$, and we denote such an $\alpha \in \mathbb{F}_{p^{2}}$ by $\sqrt{a}$. 
Theorem 5.1. Let $p \equiv 1(\bmod 4)$ be a prime and let $a, b, c$ be nonnzero elements of the field $\mathbb{F}_{p}$. Let $q$ be $p$ or $p^{2}$ according as $\chi_{p}(a c)$ is 1 or -1 , and set

$$
\gamma=\frac{b+2 \sqrt{a c}}{16 \sqrt{a c}} \in \mathbb{F}_{q} .
$$

Let $N$ be the number of $\mathbb{F}_{q}$-points on the affine curve $y^{2}=x^{4}+x^{2}+\gamma$. If $N \equiv-1(\bmod p)$, then

$$
\sum_{x \in \mathbb{F}_{p}} \chi_{p}\left(a x^{5}+b x^{3}+c x\right)=0 .
$$

For the sake of convenience, for an odd prime $p$ we introduce the following two polynomials over $\mathbb{F}_{p}$ :

$$
f(z)=1+\sum_{k=1}^{\lfloor(p-1) / 8\rfloor} \prod_{j=0}^{k-1} \frac{(8 j+1)(8 j+5)}{4(j+1)(4 j+3)} z^{k},
$$

and

$$
g(z)=1+\sum_{k=1}^{\lfloor(p-1) / 4\rfloor} \frac{(4 k-1) ! !}{4^{k}(k !)^{2}} z^{k} .
$$

Lemma 5.1. Let $p$ be a prime with $p \equiv 1(\bmod 4)$, and let $a, b, c \in \mathbb{F}_{p} \backslash\{0\}$. Define

$$
A_{p}=\sum_{x \in \mathbb{F}_{p}} \chi_{p}\left(a x^{5}+b x^{3}+c x\right) .
$$

Viewing $A_{p}(\bmod p)$ as an element of $\mathbb{F}_{p}$, we have

$$
A_{p}(\bmod p)=-\left(\begin{array}{c}
(p-1) / 2 \\
(p-1) / 4
\end{array}\right) b^{(p-1) / 4}\left(a^{(p-1) / 4}+c^{(p-1) / 4}\right) f\left(\frac{a c}{b^{2}}\right) .
$$

Consequently, $A_{p}=0$ if $\left(a^{-1} c\right)^{(p-1) / 4}=-1$ or $f\left(a c / b^{2}\right)=0$.

Proof. As $A_{p}=\sum_{x \in \mathbb{F}_{p} \backslash\{0\}} \chi_{p}\left(a x^{5}+b x^{3}+c x\right)$, we have $\left|A_{p}\right|<p$. So, the second assertion in Lemma 5.1 follows from the first one.

Now we come to prove the first assertion. With the help of (2.2), in $\mathbb{F}_{p}$ we have

$$
\begin{aligned}
A_{p}(\bmod p)= & \sum_{x \in \mathbb{F}_{p}}\left(a x^{5}+b x^{3}+c x\right)^{(p-1) / 2} \\
= & \sum_{k_{5}+k_{3}+k_{1}=(p-1) / 2} \frac{((p-1) / 2) !}{k_{5} ! k_{3} ! k_{1} !} a^{k_{5}} b^{k_{3}} c^{k_{1}} \sum_{x=0}^{p-1} x^{5 k_{5}+3 k_{3}+k_{1}} \\
= & -\sum_{\substack{k_{5}+k_{3}+k_{1}=(p-1) / 2 \\
5 k_{5}+3 k_{3}+k_{1}=p-1}} \frac{((p-1) / 2) !}{k_{5} ! k_{3} ! k_{1} !} a^{k_{5}} b^{k_{3}} c^{k_{1}} \\
& -\sum_{\substack{k_{5}+k_{3}+k_{1}=(p-1) / 2 \\
5 k_{5}+3 k_{3}+k_{1}=2(p-1)}} \frac{((p-1) / 2) !}{k_{5} ! k_{3} ! k_{1} !} a^{k_{5}} b^{k_{3}} c^{k_{1}} .
\end{aligned}
$$


(Note that if $k_{1}, k_{3}, k_{5}$ are nonnegative integers with $k_{1}+k_{3}+k_{5}=(p-1) / 2$ then $k_{1}+3 k_{3}+5 k_{5} \leqslant 5\left(k_{1}+k_{3}+k_{5}\right)<3(p-1)$.) Thus,

$$
\begin{aligned}
& A_{p}(\bmod p) \\
& =-\sum_{\substack{k_{5}+k_{3}+k_{1}=(p-1) / 2 \\
k_{3}+2 k_{5}=(p-1) / 4}} \frac{((p-1) / 2) !}{k_{5} ! k_{3} ! k_{1} !} a^{k_{5}} b^{k_{3}} c^{k_{1}}-\sum_{\substack{k_{5}+k_{3}+k_{1}=(p-1) / 2 \\
k_{3}+2 k_{1}=(p-1) / 4}} \frac{((p-1) / 2) !}{k_{5} ! k_{3} ! k_{1} !} a^{k_{5}} b^{k_{3}} c^{k_{1}} \\
& =-\sum_{k=0}^{\lfloor(p-1) / 8\rfloor} \frac{((p-1) / 2) !}{k !((p-1) / 4-2 k) !((p-1) / 4+k) !} a^{k} b^{(p-1) / 4-2 k} c^{(p-1) / 4+k} \\
& -\sum_{k=0}^{\lfloor(p-1) / 8\rfloor} \frac{((p-1) / 2) !}{((p-1) / 4+k) !((p-1) / 4-2 k) ! k !} a^{(p-1) / 4+k} b^{(p-1) / 4-2 k} c^{k} \\
& =-\left(\begin{array}{l}
(p-1) / 2 \\
(p-1) / 4
\end{array}\right) b^{(p-1) / 4}\left(c^{(p-1) / 4}+a^{(p-1) / 4}\right) \\
& \times\left(1+\sum_{k=1}^{\lfloor(p-1) / 8\rfloor} \prod_{i=0}^{2 k-1}\left(\frac{p-1}{4}-i\right) \cdot \prod_{j=1}^{k} \frac{1}{((p-1) / 4+j)} \cdot \frac{1}{k !}\left(\frac{a c}{b^{2}}\right)^{k}\right) \\
& =-\left(\begin{array}{c}
(p-1) / 2 \\
(p-1) / 4
\end{array}\right) b^{(p-1) / 4}\left(a^{(p-1) / 4}+c^{(p-1) / 4}\right) f\left(\frac{a c}{b^{2}}\right)
\end{aligned}
$$

as desired.

Lemma 5.2. Let $p$ be an odd prime and let $q=p^{n}$ with $n \in \mathbb{Z}^{+}$. For any polynomial

$$
H(x)=\sum_{k=0}^{2(p-1)} c_{k} x^{k} \in \mathbb{F}_{q}[x],
$$

we have

$$
\sum_{x \in \mathbb{F}_{q}} H(x)^{1+p+\cdots+p^{n-1}}=-c_{p-1}^{1+p+\cdots+p^{n-1}}-c_{2(p-1)}^{1+p+\cdots+p^{n-1}} .
$$

Proof. As the multiplicative group $\mathbb{F}_{q} \backslash\{0\}$ is cyclic, similar to (2.2), for each $s=0,1,2, \ldots$ we have

$$
\sum_{x \in \mathbb{F}_{q}} x^{s}= \begin{cases}-1 & \text { if } s \in(q-1) \mathbb{Z}^{+} \\ 0 & \text { otherwise }\end{cases}
$$

where we treat $0^{0}$ as 1 when $s=0$. Note also that

$$
H(x)^{p^{i}}=\sum_{k=0}^{2(p-1)} c_{k}^{p^{i}} x^{k p^{i}}
$$


for all integers $i \geqslant 0$. Thus

$$
\sum_{x \in \mathbb{F}_{q}} H(x)^{1+p+\cdots+p^{n-1}}=\sum_{x \in \mathbb{F}_{q}} \prod_{i=0}^{n-1}\left(\sum_{k_{i}=0}^{2(p-1)} c_{k_{i}}^{p^{i}} x^{k_{i} p^{i}}\right)=-\sum_{k_{0}, \ldots, k_{n-1}}^{*} \prod_{i=0}^{n-1} c_{k_{i}}^{p^{i}}
$$

where $\sum^{*}$ means that the sum is taken over all $k_{0}, \ldots, k_{n-1} \in\{0,1, \ldots, 2 p-$ 2) subject to the condition

$$
k_{0}+k_{1} p+\cdots+k_{n-1} p^{n-1} \in(q-1) \mathbb{Z}^{+} .
$$

Write $k_{i}=p-1+t_{i}$, where $-(p-1) \leqslant t_{i} \leqslant p-1$. Then

$$
\sum_{i=0}^{n-1} k_{i} p^{i}=q-1+\sum_{i=0}^{n-1} t_{i} p^{i}
$$

Note that

$$
\left|\sum_{i=0}^{n-1} t_{i} p^{i}\right| \leqslant q-1
$$

and the equality is possible only if $t_{0}=\cdots=t_{n-1}=p-1$ (i.e., $k_{0}=\cdots=$ $\left.k_{n-1}=2(p-1)\right)$ or $t_{0}=\cdots=t_{n-1}=-(p-1)$ (i.e., $k_{0}+k_{1} p+\cdots+$ $\left.k_{n-1} p^{n-1}=0\right)$. Since $\left|t_{i}\right|<p$, if $\sum_{i=0}^{n-1} t_{i} p^{i}=0$ then we obtain step by step that $t_{0}=\cdots=t_{n-1}=0$ (i.e., $k_{0}=\cdots=k_{n-1}=p-1$ ).

Combining the above, we finally obtain (5.3).

Lemma 5.3. Let $p$ be an odd prime and let $q=p^{n}$ with $n \in \mathbb{Z}^{+}$. Let $\alpha, \beta, \gamma \in \mathbb{F}_{q} \backslash\{0\}$ and set

$$
B_{q}=\sum_{x \in \mathbb{F}_{q}} \chi_{q}\left(\alpha x^{4}+\beta x^{2}+\gamma\right)
$$

Viewing $B_{q}(\bmod p)$ as an element of $\mathbb{F}_{q}$, we have

$$
B_{q}(\bmod p)=-\chi_{q}(\alpha)-\chi_{q}(\beta) g\left(\frac{\alpha \gamma}{\beta^{2}}\right)^{1+p+\cdots+p^{n-1}} .
$$

Proof. Write

$$
H(x):=\left(\alpha x^{4}+\beta x^{2}+\gamma\right)^{(p-1) / 2}=\sum_{k=0}^{2(p-1)} c_{k} x^{k} .
$$

In view of Lemma 5.2, we have

$$
\begin{aligned}
B_{q}(\bmod p) & =\sum_{x \in \mathbb{F}_{q}}\left(\alpha x^{4}+\beta x^{2}+\gamma\right)^{(q-1) / 2}=\sum_{x \in \mathbb{F}_{q}} H(x)^{1+p+\cdots+p^{n-1}} \\
& =-c_{p-1}^{1+p+\cdots+p^{n-1}}-c_{2(p-1)}^{1+p+\cdots+p^{n-1}} .
\end{aligned}
$$

Clearly,

$$
c_{2(p-1)}^{1+p+\cdots+p^{n-1}}=\alpha^{(q-1) / 2}=\chi_{q}(\alpha) .
$$


Note also that

$$
\begin{aligned}
c_{p-1} & =\sum_{\substack{k_{4}+k_{2}+k_{0}=(p-1) / 2 \\
4 k_{4}+2 k_{2}=p-1}} \frac{((p-1) / 2) !}{k_{4} ! k_{2} ! k_{0} !} \alpha^{k_{4}} \beta^{k_{2}} \gamma^{k_{0}} \\
& =\sum_{0 \leqslant k \leqslant(p-1) / 4} \frac{((p-1) / 2) !}{((p-1) / 2-2 k) !(k !)^{2}} \alpha^{k} \beta^{((p-1) / 2-2 k)} \gamma^{k} \\
& =\beta^{(p-1) / 2}+\beta^{(p-1) / 2} \sum_{k=1}^{\lfloor(p-1) / 4\rfloor} \prod_{j=0}^{2 k-1}\left(\frac{p-1}{2}-j\right) \cdot \frac{1}{(k !)^{2}}\left(\frac{\alpha \gamma}{\beta^{2}}\right)^{k} \\
& =\beta^{(p-1) / 2} g\left(\frac{\alpha \gamma}{\beta^{2}}\right)
\end{aligned}
$$

and hence

$$
c_{p-1}^{1+p+\cdots+p^{n-1}}=\chi_{q}(\beta) g\left(\frac{\alpha \gamma}{\beta^{2}}\right)^{1+p+\cdots+p^{n-1}} .
$$

Combining (5.6) with (5.7) and (5.8), we immediately obtain the desired $(5.5)$.

Now we study further properties of the polynomials $f$ and $g$ defined by (5.1) and (5.2). They may be viewed as truncated versions of certain hypergeometric series.

Lemma 5.4. Let $p$ be an odd prime and let $q=p^{n}$ with $n \in \mathbb{Z}^{+}$.

(i) A polynomial $u \in \mathbb{F}_{q}[z]$ with $\operatorname{deg} u \leqslant\lfloor(p-1) / 4\rfloor$ satisfies the differential equation

$$
\left(4 z-16 z^{2}\right) u^{\prime \prime}+(4-32 z) u^{\prime}-3 u=0
$$

if and only if $u=c g$ for some $c \in \mathbb{F}_{q}$.

(ii) Suppose that $p \equiv 1(\bmod 4)$. Then a polynomial $v \in \mathbb{F}_{q}[z]$ with $\operatorname{deg} v \leqslant\lfloor(p-1) / 8\rfloor$ satisfies the differential equation

$$
\left(16 z-64 z^{2}\right) v^{\prime \prime}+(12-112 z) v^{\prime}-5 v=0
$$

if and only if $v=c f$ for some $c \in \mathbb{F}_{q}$.

Proof. It is straightforward to verify that $u=g$ and $v=f$ satisfy (5.9) and (5.10) respectively. So, the "if" parts of (i) and (ii) are easy.

Now we prove the "only if" part of (i). If a polynomial $u \in \mathbb{F}_{q}[z]$ with $\operatorname{deg} u \leq\lfloor(p-1) / 4\rfloor$ satisfies (5.9), then there is a constant $c \in \mathbb{F}_{q}$ such that $\tilde{u}=u-c g$ is a solution of (5.9) with $\operatorname{deg} \tilde{u}<\lfloor(p-1) / 4\rfloor$. Thus, it suffices to show that (5.9) has no nonzero solution $u=c_{d} z^{d}+\cdots+c_{0}$ with $\operatorname{deg} u=d<$ $\lfloor(p-1) / 4\rfloor$. In fact, the coefficient of $z^{d}$ in $\left(4 z-16 z^{2}\right) u^{\prime \prime}+(4-32 z) u^{\prime}-3 u$ is $-(4 d+1)(4 d+3) c_{d} \neq 0$ provided $d<\lfloor(p-1) / 4\rfloor$.

Similarly, we can show the "only if" part of (ii).

Lemma 5.5. Let $p=4 n+1$ be a prime with $n \in \mathbb{Z}^{+}$. Then

$$
-(2 n) !(n !)^{2} g(z)=(16 z-2)^{n} f\left(\frac{1}{(16 z-2)^{2}}\right) .
$$


Proof. Clearly, $u=(16 z-2)^{n} f\left((16 z-2)^{-2}\right)$ is a polynomial of degree $n=(p-1) / 4$ with the leading coefficient 1. A direct computation based on (5.10) shows that $u$ satisfies (5.9). Now we apply Lemma 5.4 and compare the leading terms of both sides. Since

$$
\frac{(p-2) ! !}{4^{n}(n !)^{2}}=\frac{(p-1) !}{2^{p-1}(2 n) !(n !)^{2}} \equiv-\frac{1}{(2 n) !(n !)^{2}}(\bmod p)
$$

we immediately get the desired result.

Proof of Theorem 5.1. Since

$$
N=\sum_{x \in \mathbb{F}_{q}}\left(1+\chi_{q}\left(x^{4}+x^{2}+\gamma\right)\right)=q+\sum_{x \in \mathbb{F}_{q}} \chi_{q}\left(x^{4}+x^{2}+\gamma\right),
$$

the assumption $N \equiv-1(\bmod p)$, together with Lemma 5.3 in the case $\alpha=\beta=1$, implies that $g(\gamma)=0$. As $(16 \gamma-2)^{-2}=a c / b^{2}$, we have $f\left(a c / b^{2}\right)=0$ by Lemma 5.5. Applying Lemma 5.1 we obtain the desired result.

\section{REFERENCES}

[1] B. C. Berndt, R. J. Evans and K. S. Williams, Gauss and Jacobi Sums, Wiley, New York, 1998.

[2] R. Chapman, Determinants of Legendre symbol matrices, Acta Arith. 115 (2004), 231-244.

[3] D. A. Cox, Primes of the Form $x^{2}+n y^{2}$, John Wiley \& Sons, 1989.

[4] K. Ireland and M. Rosen, A Classical Introduction to Modern Number Theory, 2nd Edition, Grad. Texts. Math., vol. 84, Springer, New York, 1990.

[5] R. Lidl and H. Niederreiter, Finite Fields, 2nd Edition, Encyclopedia of Math. and its Applications, 20, Cambridge Univ. Press, Cambridge, 1997.

[6] A. R. Rajwade, The Diophantine equation $y^{2}=x\left(x^{2}+21 D x+112 D^{2}\right)$ and the conjectures of Birch and Swinnerton-Dyer, J. Austral. Math. Soc. Ser. A 24 (1977), 286-295.

[7] Z.-H. Sun, Congruences involving Legendre polynomials II, J. Number Theory 133 (2013), 1950-1976.

[8] Z.-W. Sun, Super congruences and Euler numbers, Sci. China Math. 54 (2011), 2509-2535.

[9] Z.-W. Sun, Supercongruences involving products of two binomial coefficients, Finite Fields Appl. 22 (2013), 24-44.

[10] Z.-W. Sun, A series of conjectures on $\sum_{x=0}^{(p-1) / 2}\left(\frac{x^{5}+c x^{3}+d x}{p}\right)$ (III), Question 319259 on MathOverflow, December 22, 2018. Available from https://mathoverflow.net/questions/319259,

[11] Z.-W. Sun, On some determinants with Legendre symbol entries, Finite Fields Appl. 56 (2019), 285-307.

[12] M. Vsemirnov, On the evaluation of R. Chapman's "evil determinant", Linear Algebra Appl. 436 (2012), 4101-4106.

[13] M. Vsemirnov, On R. Chapman's "evil determinant": case $p \equiv 1$ ( $\bmod 4)$, Acta Arith. 159 (2013), 331-344. 
(Dmitry Krachun) St. Petersburg Department of Steklov Mathematical Institute of Russian Academy of Sciences, Fontanka 27, 191023, St. PetersBURG, RUSSIA

E-mail address: dmitrykrachun@gmail.com

(Fedor Petrov) St. Petersburg Department of Steklov Mathematical Institute of Russian Academy of Sciences, Fontanka 27, 191023, St. Petersburg, RUSSIA

E-mail address: fedyapetrov@gmail.com

(Zhi-Wei Sun) Department of Mathematics, Nanjing University, Nanjing 210093, People's Republic of China

E-mail address: zwsun@nju.edu.cn

(Maxim Vsemirnov) St. Petersburg Department of Steklov Mathematical Institute of Russian Academy of Sciences, Fontanka 27, 191023, St. PetersBURG, RUSSIA

E-mail address: vsemir@pdmi.ras.ru 